\title{
COLLISIONAL-DISSOCIATIVE RECOMBINATION OF \\ ELECTRONS WITH MOLECULAR IONS
}

by

C. B. Collins

Southwest Center for Advanced Studies
Dallas, Texas

Submitted to The Physical Review

$$
\text { March, } 1965
$$

\section{UNPUBLLISIDD PREIMINARY DATA}

GPO PRICE $\boldsymbol{s}$

This work was supported by CFSTI PRICE(S) NASA Grant NsG-262-69 264
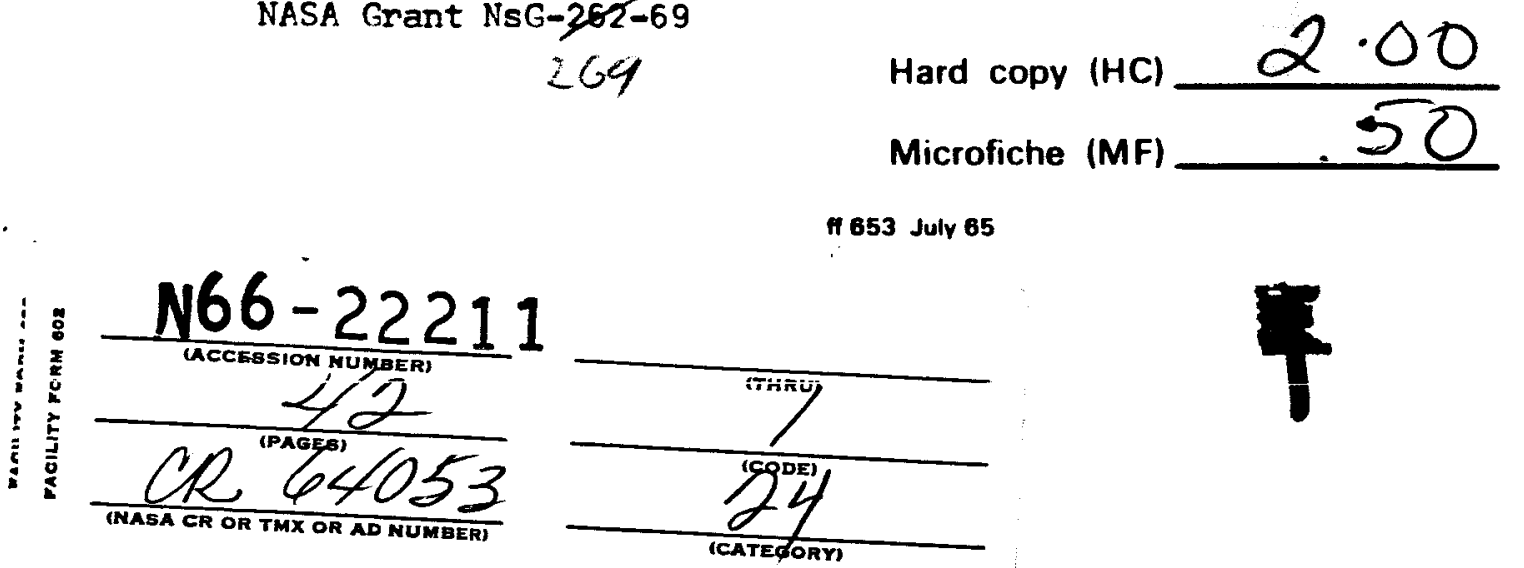


$$
22211
$$

The recombination rate of electrons in a hypothetical plasma containing ions of a molecule having both repulsive and bound neutral states is calculated for electron densities between $10^{10}$ and $10^{14} / \mathrm{cm}^{3}$ and temperatures ranging from $250^{\circ} \mathrm{K}$ to $2000^{\circ} \mathrm{K}$. It is found that if the molecule has a dissociative state located sufficiently close in energy to the molecular ion, values are obtained considerably in excess of the collisional-radiative recombination rate for systems possessing only bound states.
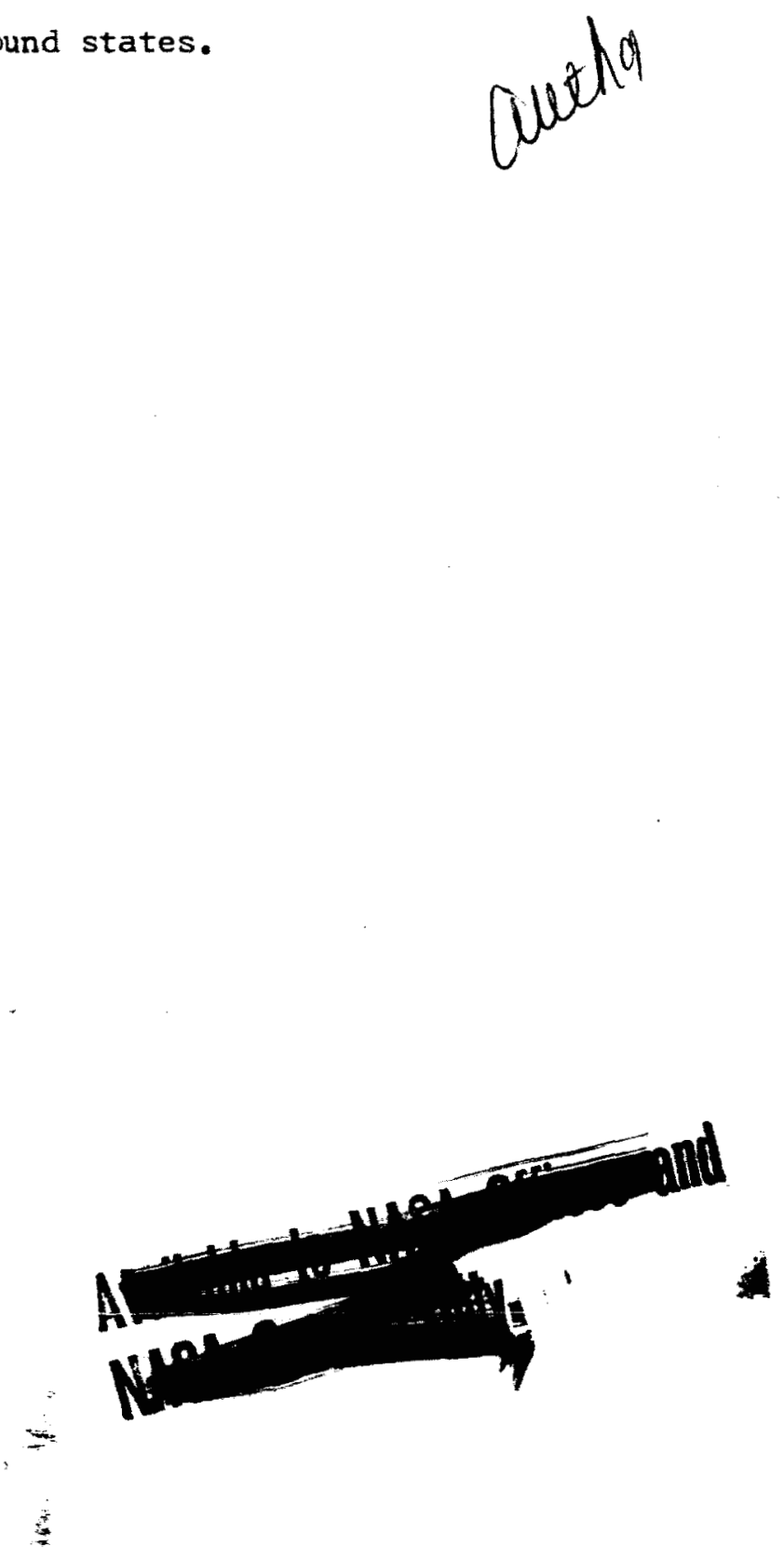


\section{INTRODUCTION}

Dissociative recombination ${ }^{1}$ of molecular ions with electrons requires a "crossing" between the potential curves of the rolecular ion and some repulsive state of the neutral molecule to explain certain large observed electron removal rates observed in decaying plasmas ${ }^{2}$. However, the necessity of such a crossing can be avoided by including into the scheme of collisional-radiative recombination, ${ }^{3}$ as applied to molecular ions, one or more repulsive states lying energetically below the molecular ion at all internuclear separations. Such a level could be expected to be populated at a rate comparable to that for an enerpetically equivalent bound state. However, re-ionization would be substentially less probable since dissociation would occur, raising the energy necessary for subseouent re-ionization of the products by at least an amount equal to the dissociation energy of the molecular ion. Since the net rate of electron removal can be considered to be the gross rate of removal due to two-electron and radiative recombination less the total rate of re-ionization of resultant excited states, a diminution of the latter as described would result in an increase in the net electron removal rate. This paper presents a mathematical model of such a collisionaldissociative process together with quantitative results. 
THEORY

In order to facilitate computation while preserving generalized properties common to a variety of real plasmas, the calculations discussed in this paper were performed on a plasma consisting of ions of a rather idealized molecule. In the initial calculations the following properties were incorporated into the molecular model:

1. a substantial dissociation energy for the molecular ion, at least several times the thermal enerpy of the electron gas,

2. a sequence of potential curves representing the possible bnund electronic states of the molecule having minima at similar internuclear separations,

3. a hydrogenic spacing in energy of the vibrationless ground states of each electronic potential curve, and

4. population only in the vibrationless ground state of each electronic state. Such a system, although highly artificial hes many common properties with the group of diatomic molecular systems which permit a united-atom description, such as molecular hydrogen and heliur.

The possibility of spontaneous dissociation was introduced in two manners. The first and simplest was used in the majority of calculations. Subsequently refinements were introduced to bring the system into a closer approximation to real molecular systems.

In the simplest, all the bound states for a particular hydrogenic energy level are replaced by the same number of dissociating states energetically equivalent to the bound states at the internuclear separation of the latter. 
Figure 1 illustrates how such a system might be represented, together with a derived energy level diagram. In this particular example it is the third electronic level which has been made dissociative.

Refinements consisted of introducing a single dissociating state having an energy which interpolated between the hydrogenic values at the equilibrium internuclear separation of the bound states. As will be presented in the results, such a refinement was found to vary the electron recombination rates subsequently calculated only moderately while serving, to introduce two specialized additional parameters which could not be easily determined, were a comparison to be made to a real decaying plasma.

of primary interest to be derived from a study of this model plasma is the value of the rate at which electrons are lost from the plasma by recombination with the molecular ions.

As in the theory of collisional-radiative recombination of protons and electrons ${ }^{3}$ the time rate of change of the population of the $i^{\text {th }}$ electronic state in a decaying plasma is given by the following expression:

$\frac{d N_{i}}{d t}=\alpha N^{+} \mathrm{Ne}+\sum_{j>i} A_{i j} N_{j}+N_{e} \sum_{j \neq i} O_{i j} N_{j}-N_{i}\left[\sum_{j<i} A_{j i}+N_{e} \sum_{j \neq i} O_{j i}+B_{i}\right]$ where $\mathrm{N}_{j}, \mathrm{~N}^{+}$and $\mathrm{N}_{e}$ are the populations of the $j^{\text {th }}$ excited state, ion density and electron density, respectively, $A_{i j}$ and $\left(Q_{i j}{ }^{N}\right)$ are the transition rates from the $j^{\text {th }}$ to the $i^{\text {th }}$ level by spontaneous radiation and inelastic or superelastic collisions with free electrons, respectively, $a$ is the coefficient for recombination from the continuum to the $i^{\text {th }}$ level by radiative and two electron recombination, and $B_{i}$ is the rate coefficient for ionization of the 
$i^{\text {th }}$ level. If the rate at which individual molecules in the $i^{\text {th }}$ state are lost, as given by the bracketed term, is large compared to the rate at which the electron density is decreasing, then $N_{i}$ can be expected to decay rapidly to a quasi-equilibrium value satisfying equation (1) when the left side is set equal to zero. This can be expected for all levels having $i>1$ and consequently an infinite set of equations linear in the $\mathrm{N}_{j}$ 's is obtained. For manipulation the set can be made finite by considering the hydrogenic sequence of states to have a greatest quantum. number $m$ above which there is a fap and the continuum of free states. As a desired quantity $\mathrm{Z}_{\mathrm{m}}$ is obtained for this particular finite set, $m$ can be increased until $\Delta \mathrm{z}_{\mathrm{m}} \cong 0$ for $m$ greater then some interger $k$. In this case $z_{m}$ can be considered to have converged to a value $\mathrm{Z}$ characteristic of the infinite set.

Numeric values for the coefficients needed in equation (1) can be obtained by calculating ${ }^{4}$ the $A_{i j}$ 's, using Bates' values ${ }^{5}$ for the radiative recombination part of $\alpha \mathrm{N}^{+} \mathrm{N}_{e}$ and calculating the $Q_{i j}, B_{i}$, and collisional fart of $\alpha N^{+} \mathrm{N}_{e}$ by Gryzinski's method ${ }^{6}$. In these latter calculations only a hydrogenic statistical weight, characteristic of the electronic degeneracy, ecual to $2 j^{2}$ for the $j^{\text {th }}$ level, was used. This is consistent with the assumption of common equilibrium internuclear separations of the various states since approximately eoual rotational partition functions would result and subsequently cancel.

Dissociation of the $\mathrm{q}^{\text {th }}$ state is represented by an additional term in the $q^{\text {th }}$ equation of

$$
-A_{D} N
$$

where $A_{D}$ is the rate coefficient for spontaneous dissociation, typically on the order $10^{13}-10^{14} / \mathrm{sec}$. Choosing a vaiue for the independent variatile 
$\mathrm{N}_{\mathrm{e}}$ and requiring

$$
\mathrm{N}^{+}=\mathrm{N}_{\mathrm{e}},
$$

Gaussian substitution was employed to reduce the set of $\mathrm{m}-1$ linear equations to a set of relations of the form

$$
N_{i}=a_{i}+b_{i} N_{1}
$$

where $i>1, a_{i}$ and $b_{i}$ are derived coefficients which are functions of the value of $\mathrm{N}^{+}$and $\mathrm{N}_{\mathrm{e}}$ chosen, and $\mathrm{N}_{1}$ is the ground state population. The electron removal rate can now be obtained by first taking the derivative of ( 3 )

$$
\frac{d N}{d t}=\frac{d N^{+}}{d t}
$$

Conservation of material requires

$$
N^{+}+\left(\sum_{i \geq 2} N_{i}\right)+N_{1}+N_{0}=C,
$$

where the summed term represents the total population of excited states and $\mathrm{N}_{0}$ is the population of all atomic states resulting from the dissociation of the $q^{\text {th }}$ level and subsequent collisional radiative processes. The derivative of $(6)$ is

$$
\frac{d N^{+}}{d t}+\frac{d}{d t}\left(\sum_{i \geq 2} N_{i}\right)+\frac{d N_{1}}{d t}+\frac{d N_{0}}{d t}=0 .
$$

Whenever a quasi-equilibrium has been established and the total population of excited states is small in comparison with the charge densities, term two in equation (7) can be neglected. Consequently combining (5) and (7) 


$$
\frac{d N_{e}}{d t}=-\left[\frac{d N_{1}}{d t}+\frac{d N_{0}}{d t}\right] \text {. }
$$

The two terms on the right can be considered to be a collisional-radiative recombination rate and a collisional-dissociative recombination rate respectively. The term $\mathrm{dN}_{\mathrm{o}} / \mathrm{d} t$ must equal the gain of atomic states by dissociation ${ }_{D} \mathrm{~N}_{\mathrm{q}}$ less the re-ionization rate of these states. The latter will be neglected since its inclusion would require a substantial number of specialized assumptions about the particular form of the dissociating level and the sequence of atomic states subsequently populated. However, since the energy required for ionization is larger after the dissociation by an amount equal to the repulsive energy of the dissociating state plus the dissociation energy of the molecular ion, for a strongly bound ion this term will generally be negligable at all but the high values of electron temperatures. Consequently

$$
\frac{d N_{O}}{d t}=A_{D} N_{q}
$$

Rewriting this as the product of a collisionel-dissociative rate coefficient, $a_{D}$. and the charge densities gives

$$
\frac{d N}{d t}=a_{D} N^{+} N_{e}={ }^{A} D^{N},
$$

or substituting from (4) where $i=q$

$$
a_{D}=\frac{A_{D^{a}}}{N^{+} N_{e}}+\frac{A_{D}^{b} g}{N^{+} N_{e}} N_{1}
$$

Similarly the collisional-radiative recombination rate of electrons, -dil $1^{\prime}$ dt can be defined in terms of a rate coefficient, * ${ }$, as follous: 


$$
\frac{d N}{d t}=a_{C R} N^{+} N_{e}
$$

and computed as follows by substituting (4) into equation (1) with $i=1$ :

$$
\begin{aligned}
a_{C R} & =\frac{1}{N^{+} N_{e}}\left\{a N^{+} N_{e}+\sum_{j=2}^{k}\left(A_{1 j}+Q_{1 j} N_{e} a_{j}\right.\right. \\
& \left.+\left[\sum_{j=2}^{k}\left(A_{1 j}+Q_{1 j} N_{e}\right)_{j}-\sum_{j=2}^{k} N_{e} Q_{j l}-B_{1}\right] N_{1}\right\} .
\end{aligned}
$$

Then

$$
\frac{d N_{e}}{d t}=-\left(\alpha_{C R}+a_{D}\right) N^{+} N_{e}
$$


RESULTS

In practice it was found that for electron temperatures in the range $250^{\circ}$ to $2000^{\circ} \mathrm{K}$ and electron densities varying from $10^{10}-10^{14} / \mathrm{cm}^{3}$, from 30 to 50 levels were required for the populations of the various excited states to converge to values which could be considered to be independent of $\mathrm{m}$, the number of bound levels used in the calculations. Similar results were found for the rate coefficients, such as $\alpha_{C R}$, dependent on the populations. Fig. 2 illustrates this with the example of the collisional-radiative rate coefficient $\alpha_{C R}$ for recombination of hydrogenic ions with electrons in the absence of dissociating levels ${ }^{7}$. As could be expected the number of levels required for convergence of the various populations and recombination coefficients calculated depended strongly and inversely on the electron temperature as well as the electron density. For electron densities between $10^{10}$ and $10^{14} / \mathrm{cm}^{3}$, 50 levels were used for an electron temperature of $250^{\circ} \mathrm{K}, 40$ for $500^{\circ} \mathrm{K}$ and 30 for higher temperatures.

Subsequent calculations of the population coefficients $a_{i}$ and $b_{i}$ as defined by equation (4) revealed that over the range of electron temperatures and densities examined empirically,

$$
a_{i}>\left|b_{i}{ }_{1}\right|,
$$

for reasonable values of $N_{1}\left(10^{17} / \mathrm{cm}^{3}\right)$ which could be expected to be found in afterglow plasmas. Physically this implies that the population of the excited levels are little affected by re-excitation of ground state molecules at temperatures not exceeding $2000^{\circ} \mathrm{K}$.

Applying (15) to equation (11) simplifies the calculation of the collisionaldissociative recombination coefficient, $\alpha_{D}$, yielding 


$$
\alpha_{D}=\frac{{ }^{A} D^{a} g}{N^{+} N_{e}}
$$

Using a spontaneous dissociation coefficient for the $o^{\text {th }}$ level of

$$
A_{D}=10^{13} / \mathrm{sec}
$$

values for $\alpha_{D}$ were calculated for electron temperatures of $250^{\circ} \mathrm{K}-2000^{\circ} \mathrm{K}$, electron densities between $10^{10}$ and $10^{14} / \mathrm{cm}^{3}$, and a variety of q's. Figure 3 ; $a, b, c$, and $d$ shows typical behavior of $\alpha_{D}$ as a function of electron density, $\mathrm{N}_{e}$ and parametrically as a function of $\mathrm{q}$ for temperatures between $250^{\circ} \mathrm{K}$ and $2000^{\circ} \mathrm{K}$. The collisional-radiative recombination rate $\alpha_{C R}$ for non-dissociating molecules is shown for comparison. It can be seen that for dissociation at a sufficiently high quantum level, the collisional-dissociative recombination rate can substantially exceed the regular collisional-radiative rate for a non-dissociating molecule.

of greater importance is the total recombination rate as defined by equation (14) or

$$
\alpha_{\text {TOTAL }}=\alpha_{D}+\alpha_{C R}
$$

The $a_{C R}$ appearing in this equation will not in general be the same as the $a_{C R}$ for a non-dissociating molecule such as shown in Fig. 3; but rather the $a_{C R}$ obtained by substituting the $a_{i}$ and $b_{i}$ obtained from the set of $m$ equation describing the dissociating system and used to calculate $\alpha_{D}$ into equation (1). Over the range of parameters considered the bracketed term in equation (13) is completely negligible owing to relation (15) and extremely small values of $Q_{j l}$ and $B_{I}$. Consequently, for evaluation $\alpha_{C R}$ can be written 


$$
\alpha_{C R}=\frac{1}{N^{+} N_{e}}\left[a N^{+} N_{e}+\sum_{j=2}^{k}\left(A_{1 j}+O_{1 j} N_{e}\right)_{j}\right] \text {. }
$$

This collisionat radiative coefficient is always substantially smaller than the corresponding coefficient for a non-dissociating system.

Figure $4 ; a, b, c$, and $d$ present the results of the calculations of the total electron removal rate by recombination with molecular ions possessing one hydrogenically spaced dissociating level of $2 q^{2}$ states. The collisionalradiative rate $\alpha_{C R}$ for the same system without the dissociating level is shown for comparison.

Several important features should be noted. The first is that each curve in Figures $4 a, b, c$, and $d$ approaches a straight line with unit slope as the electron density is increased. Although the same is true of the $\alpha_{C R}$ for a non-dissociating system, the inclusion of a dissociating level at markedly enchances this effect. Consequently if

$$
\mathrm{N}^{+}=\mathrm{N}^{-}
$$

then the loss of electrons by recombination in such a system will be third order in the charge density over a wider range of electron densities than in a system including no dissociating molecular level.

A second observation which can be made is that although the dependence of $a_{D}$ on the electron density is considerably more complex as shown in Figures $3 a, b, c$, and $d$, under certain conditions, large ranges of linearity exist in the $\log \alpha_{D}$ versus $\log N_{e}$ plots. The slope again is unity implying that, assuming $(20)$, the rate of production of atoms by the dissociation of 
the $\mathrm{q}^{\text {th }}$ molecular level is third order in the charge density under these conditions. Using terms previously defined, this can be written

$$
\frac{\mathrm{dN}_{\mathrm{O}}}{\mathrm{dt}}=\mathrm{CN}_{\mathrm{e}}^{3}
$$

where $C$ is a function only of $q$ and the electron temperature.

Finally the detailed behavior of the electron removal rate, as the quantum level of dissociation is varied should be noted. It was found, as can be seen from Figures $4 a, b, c$ and $d$, that the total electron, and consequently ion, removal rate, $\alpha_{\text {TOTAL, }}$ is never less than the collisional-radiative rate for a non-dissociating system under the same conditions. Instead, for dissociation at sufficiently low quantum levels, $q$, the two rates are approximately equal. This results from the fact that the total electron removal rate can be considered to be the gross rate at which electrons are removed by recombining events less the rate of reionization of the resultant bound states. Since, for the system outlined for study here, the former is unaffected by the presence or absence of dissociating states of the neutral molecule, the net electron removal rate can be increased only by a diminution of the rate of re-ionization of excited bound states.

For moderate temperatures and densities studied here, sufficiently low quantum levels exist from which re-ionization and re-excitation to more readily ionized levels is negligible. Since all the electrons in such a level are "effectively catpured", any further reduction in the rate of reionization or re-excitation of these levels by dissociation will have a negligible effect on the total rate at which free electrons are produced by re-ionization. Figure $5 \mathrm{a}$, which shows the excited state populations both with and without dissociation at $q=4$ at $2000^{\circ} \mathrm{K}$ and $10^{13}$ electrons $/ \mathrm{cm}^{3}$, in 
comparison with Figure 4d illustrates this effect. Introduction of a dissociating level at $q=4$ serves to depress only the populations of the fourth and lower-lying quantum levels. Since higher-lying levels are not appreciably depressed, re-excitation of the fourth quantum level can be neglected and consequently, re-ionization from it, as the latter is energetically even less feasible. It could thus be expected that the total rate of re-ionization and consequently, the net electron removal rate would be unaffected by the dissociation. Figure 4d verifies this expectation showing $\alpha_{\text {TOTAL }}$ to be indistinguishable from $\alpha_{C R}$, calculated in the absence of dissociation. Conversely, if the level, $a$, at which dissociation occurs is sufficiently high that substantial re-ionization results from it or if the introduction of dissociation at $q$ has served to depress the populations of higher lying levels indicating that re-excitation from $q$ to higher lying levels was not negligible, the net electron removal rate could be expected to be significantly increased by virtue of a substantial diminution of the rate at which free electrons are produced by re-ionization. Figure $5 \mathrm{~b}$, which presents the case in which dissociation occurs at $q=6$, illustrates the depression of the higherlying levels, and Figure 4d the resulting increase in the net electron removal rate $\boldsymbol{\alpha}_{\text {TOTAL }}$

Inherent in the determinations of $\alpha_{D}$ and $\alpha_{\text {TOTAL }}$ under the various conditions examined has been the necessity of assuming a value for $A_{D}$, the coefficient of spontaneous dissociation for the dissociating level. As stated in equation (17) the value used in these calculations was $10^{13} / \mathrm{sec}$. However, the preceeding analysis of the detailed mechanism which attributes the increase in $\alpha_{\text {TOTAL }}$ to a reduction of the rates of re-ionization and re-excitation of the $q^{\text {th }}$ level caused by the competing process of dissociation suggests that 
a type of saturation should occur whenever its rate coefficient, ${ }_{D}$, greatly exceeded the coefficients for other loss mechanisms from the level, such as superelastic collisions with electrons. In this case essentially all of molecules in the $q^{\text {th }}$ state would dissociate and a further increase in $A_{D}$ would yield no greater production of atomic species since the rate would then be limited by the rate at which electrons entered the $q^{\text {th }}$ level. Figure S presents typical results verifying the general absence of a dependence of the collisional-dissociative recombination rate $\alpha_{D}$ on the spontaneous dissociation rate $A_{D}$, at $2000^{\circ} \mathrm{K}$ and $10^{13}$ electrons $/ \mathrm{cm}^{3}$. Only the slightest variation can be seen for $q=18$ and $A_{D}=12^{12} / \mathrm{sec}$, conditions under which spontaneous dissociation comprises only about $90 \%$ of the total loss rate from the 18th level. Dissociation at lower $q$ 's, for which $A_{D}$ is orders of magnitude greater than the loss rate by other processes, shows no appreciable dependence of $\alpha_{D}$ on $A_{D}$. Since the total non-dissociative loss rate from these higher rates is principally by superelastic collisions with electrons, a process having a small rate dependence on temperature, results at lower temperatures should differ little from those shown in Figure 5 . Consequently $A_{D}$ need not be considered a significant parameter for these calculations as long as it is of the order of a spontaneous dissociation rate, $10^{13} / \mathrm{sec}$.

Examination of the results presented in Figures 3 and $4: a, b, c$, and d reveal no simple dependence of either $\alpha_{D}$ or $\alpha_{\text {TOTAL }}$ on electron temperature. Only the general observation can be made that $\alpha_{D}$ has a weaker dependence on electron temperature than the normal collisional radiative rate for nondissociating systems. This is evidenced by the fact that for dissociation at any particular level $q$, the ratio of $\alpha_{D}$ to the $\alpha_{C R}$ characteristic of a nondissociating system decreases with decreasing temperature. 
Complications in applying these results to real molecular systems rest in certain simplifications introduced into the basic model to represent the dissociation. Thus far, as discussed previously, it has been assumed all $2 q^{2}$ states of a dissociative level, $q$, dissociated, when in a real system a mixture of bound and dissociative states might be included in a particular level. Even more probable is that the energy of a dissociating state at the appropriate intemuclear separation will lie between the hydrogenic energies of the bound levels. Consequently, a modification of the original model was considered having the following properties:

1. the dissociating state or states introduced are represented by a level on the energy level diagram having a quantum defect, or a non-integral effective principal quantum number, and

2. the statistical weight of the dissociating state is introduced as an independent variable.

Using this model, values for $a_{D}$ and $a_{\text {TOTAL }}$ were calculated. 'It was found that in most cases neither the interpolation of the effective quantum number nor a degeneracy of unity rather than the $2 q^{2}$ of the previous model caused variations outside the range of arbitrariness inherent in the inelastic cross-sections employed in the computations, a factor of two or three. Generally with a unit statistical weight the values of $a_{\text {TOTAL }}$ for interpolated quantum levels, $q^{\prime}$, were found to continuously approach the values obtained for complete dissociation of all states of the $q^{\text {th }}$ level as $q^{\prime}$ approached the integral $q$.

The exceptions occurred under the combination of high quantum number of dissociation and high electron density. Under these conditions the total electron removal rate, dominated by $\alpha_{D}$, is theoretically found to pass to an 
asymptotic form dependent on both $A_{D}$, and the number of states assumed dissociating. Consequently at these higher quantum levels $\alpha_{D}$ should approach a substantially lower value as $q^{\prime}$ approaches $q$. Figure 7 illustrates both types of behavior as $q^{\prime}$ approaches $q$ for the case of a $2000^{\circ} \mathrm{K}$ plasma containing $10^{13}$ electrons $/ \mathrm{cm}^{3}$.

The asymptotic form of $a_{D}$ for high quantum levels can be derived by writing it in the form

$$
\alpha_{D}=\frac{{ }_{D} N_{g}}{N^{+} N_{e}}
$$

For sufficiently high levels that the re-ionization of the $q^{\text {th }}$ level is greater than the net unbalanced downward loss rates including dissociation, then the $q^{\text {th }}$ level will be in Saha equilibrium with the free electrons and the population will be given by

$$
N_{Q}=\frac{g_{q}}{g^{+} g^{-}}\left(\frac{2 \pi m K T_{e}}{h^{3}}\right)^{-3 / 2} e^{I q / k T} e N^{+} N_{e}
$$

where $g_{q}, g^{+}$and $g^{-}$are the statistical weights of the dissociating level, ion, and free electron respectively and $\mathrm{Iq}$ is the ionization potential of the $q^{\text {th }}$ state, other terms having conventional meanings. Substituting (23) in (22) and letting $\mathrm{Iq} \rightarrow 0$,

$$
a_{D}=\frac{g_{g}}{g^{+} g^{-}} A\left(\frac{2 \pi m k T}{h^{3}}\right)^{-3 / 2}
$$

Figure 7, shows that for the case of a single dissociating state $\alpha_{D}$ is approaching the asymptotic limit for large $?$; calculated by $(24)$, of $2.33 \times 10^{-8}$ $\mathrm{cm}^{3} / \mathrm{sec}$ under these conditions. 
At the lower levels of dissociation it can be seen that the value of $\alpha_{D}$ for dissociation of a single interpolated level, $q^{\prime}$ should be continuous with the value for dissociation of all $2 q^{2}$ states of the next lowest integral level $q$ by considering that the $q^{\prime}$ state must pass to a limiting case as $q^{\prime}$ approaches $q$ in which it is but one of the degenerate states of the $q^{\text {th }}$ level. Writing equation ( 1 ) for the $q^{\text {th }}$ level containing the $q^{\prime}$ state, denoting the gain terms by $G$, and including the dissociative loss

$$
\frac{d N_{q}}{d t}=G-N q\left[N_{E} \sum_{j \neq q}^{k} Q_{j q}+\sum_{j<q}^{k} A_{j q}+B q\right]-A_{D^{N} q^{\prime}}
$$

As a degenerate sublevel the population $\mathrm{N}_{\mathrm{q}}$, can be written

$$
N_{q^{\prime}}=\frac{1}{2 q^{2}}{ }_{q}
$$

which when substituted into (25) and collecting terms gives

$$
N_{q}=\frac{G}{\left[N_{e} \sum_{j \neq q}^{k} Q_{j q}+\sum_{j<q}^{k} A_{j q}+B_{q}\right]+A_{D} / 2 q^{2}}
$$

For terms having sufficiently low $q$ that the term in brackets is negligible compared to the last term in the denominator,

$$
N_{Q}=\frac{2 q^{2} G}{A_{D}}
$$

and from (26)

$$
N_{q^{\prime}}=\frac{G}{A_{D}}
$$


Consequently the rate at which electrons are captured by the collisional dissociative process is

$$
\frac{d N_{0}}{d t}=A_{D} N_{q^{\prime}}=G
$$

the rate at which molecules enter the $q^{\text {th }}$ level.

For dissociation of all $2 \mathrm{q}^{2}$ states the $\mathrm{N}_{\mathrm{q}^{\prime}}$ in equation (25) is replaced by $\mathrm{N}_{\mathrm{q}}$ leaving the bracketed term in the denominator of (27) to be compared with $A_{D}$ rather than $A_{D} / 2 q^{2}$. Since the bracketed terms are virtually the same for both cases, they can be neglected for larger values of $q$ in the case of dissociation of all $2 q^{2}$ states giving

$$
\mathrm{N}_{\mathrm{q}}=\frac{\mathrm{G}}{\mathrm{A}_{\mathrm{D}}}
$$

and

$$
\frac{d N}{d t}=A_{D} N_{q}=G
$$

In comparison with (30), equation (32) establishes the continuity for the two cases as $q^{\prime}$ approaches $q$ provided that $q$ is sufficiently small that the bracketed loss term in the denominator of (27) is negligible in comparison with $A_{D} / 2 q^{2}$ and consequently, $A_{D}$, so that equations (30) and (32) are applicable.

In practice, provided neither $\mathrm{q}$ nor $\mathrm{N}_{e}$ is too large, little error would be introduced in considering the value of $\alpha_{\text {TOTAL, characteristic of a }}$ molecular system containing a single non-degenerate repulsive state with an effective non-integral quantum number $q^{\prime}$, to be equal to the $\alpha_{\text {ToTAI }}$ for the 
simple case of total dissociation of the $q^{\text {th }}$ hydrogenic level as shown in Figures $4 ; a, b, c$, and $d$ and where $q$ is chosen to be the largest interger less than $q^{\prime}$. Detailed verification of this approximation was conducted and Figure $4 ; a, b, c$, and $d$ present only values of $q$ which are sufficiently low that use of this approximation would introduce error less than a factor of 2.5 for dissociation through states having non-intergral principal quantum numbers and multiplicity not exceeding $2 q^{2}$.

It should be noted that were the detailed potential curves of the molecule completely known, in the case of a single dissociating state the fiction of the effective non-integral quantum number of this level would be replaced by a finite group of effective quantum numbers representing sections of the repulsive curve which were consequently functions of the internuclear separation, each with its associated $A_{D}$ incorporating the appropriate overlaps integral. Application of a limiting process in which the repulsive curve was divided successively finer would yield a more realistic $\alpha_{D}$. However considering the insensitivity of $\alpha_{D}$ of $A_{D}$ at low quantum levels as well as to the exact energy of the dissociating level suggests that such a process would yield results varying little from those of figure $4 ; a, b, c$, and $d$. 


\section{DISCUSSION}

As discussed previously, large electron-ion recombination rates have been observed in gases capable of forming molecular ions ${ }^{2}$. Prior interpretations relied upon dissociative recombination ${ }^{1}$ of the molecular ions with difficulty in some instances, notably helium ${ }^{8}$. Collisional-dissociative recombination offers a possible alternative explanation since it must occur to some extent for all gases capable of forming molecules having one or more dissociative states energetically below the ionization limit. The question of degree is the factor determining whether or not collisional-dissociative recombination need be considered as an alternative to dissociative recombination and collisional-radiative recombination of the molecular ions formed.

The theoretical treatment conducted here gives an indication of the degree of importance of collisional-dissociative recombination of electrons with molecular ions. Certainly at higher electron densities in all plasmas containing molecular systems possessing high-lying repulsive states, it is a necessary consideration. For those gases, such as hydrogen and helium, whose molecular states approximate united-atom conditions, thus possessing hydrogenically spaced energy levels, the best agreement with the theory of collisional-dissociative recombination could be expected.

Molecular helium, in particular, Dossesses states whose energy spacing is quite a good approximation to hydrogenic above the third level. In addition the molecular ion is strongly bound and the principal population of each state apparently lies in the vibrationless ground state of each level as evidenced by spectroscopic data. Since, in addition the excited states have approximately equal equilibrium internuciear separation ${ }^{9}$, mólecülār helium is realistically 
approximated by the idealized molecule of this study ${ }^{10}$. Although no repulsive states are known to exist for molecular helium, other than the ground state which would not effectively increase the electron removal rate, conversely not all possible states have been spectroscopically located.

Recent spectroscopic measurements ${ }^{11}$ of the $10,830 \AA$ helium line emitted by the $2^{3} \mathrm{P}+2^{3} \mathrm{~S}$ transition in atomic helium in a recombining helium plasma have revealed that a majority of the $2^{3} \mathrm{p}$ atoms observed at the higher pressures $(\sim 30 \mathrm{~mm} \mathrm{Hg}$.$) result from a recombination of \mathrm{He}_{2}^{+}$. Absolute intensity measurements of the $10,830 \AA$ emission were used to establish that the recombination coefficient characteristic of the process by which the molecular ions were recombining and resulting in the subsequent production of neutrai atoms was

$$
\alpha_{D} \leq 2 \times 10^{-11} \mathrm{~cm}^{3} / \mathrm{sec}
$$

at $1800^{\circ} \mathrm{K}$ and $2 \times 10^{12}$ electrons $/ \mathrm{cm}^{3}$.

Whereas this value is quite small to be interpreted by dissociative recombination, comparison with Figure $3 d$ shows that collisional-dissociative recombination through a level near $q=4$ could be expected to given an electron removal rate of

$$
\alpha_{D}=10^{-11} \mathrm{~cm}^{3} / \mathrm{sec} \text { at } 2000^{\circ} \mathrm{K}
$$

Consequently a dissociative state having an energy at the common equilibrium internuclear separation of the bound molecular states comparable to or below that of the fourth hydrogenic level would yield a collisional-dissociative recombination rate in agreement with that experimentally determined. Since the states with one of the possible $\Lambda$-values arising from the combination $\mathrm{He}\left(1^{1} \mathrm{~S}\right)+$ $H\left(2^{3} \mathrm{P}\right)$, the $4 \mathrm{p} \Sigma$, has not been experimentally located, such a repulsive state with 
the desired energy could exist. Consequently at least on the basis of recombination rates, collisional-dissociative recombination offers a promising altemative to dissociative recombination in the explanation of the dissociation observed in decaying helium plasmas. 


\section{ACKNOWLEDGMENT}

This research has been supported by the National Aeronautics and Space Administration under grant No. NsG-269-62. 


\section{REFERENCES}

1. D. R. Bates, Phys. Rev. 77, 718 (1950); 78, 492 (1950); 82, 103 (1951).

2. These large rates are found only in plasmas known to form an abundance of molecular ions; see L. B. Loeb: Handbuch der Physics, edited by S. Flugge (Springer-Verlag, 1956), $\mathrm{p}=490$.

3. D. R. Bates, A. E. Kingston, and R. W. P. McWhirter, Proc. Roy. Soc. A267. 297 (1962).

4. L. C. Green, P. P. Rush, C. D. Chandler, Astrophys. J. Suppl. 3, 37 (1957).

5. D. R. Bates and A. Dalgarno, in Atomic and Molecular Processes edited by D. R. Bates (Academic Press Inc., New York, 1962), p. 249. 6. As discussed by Kingston: A. E. Kingston, Phys. Pev. 35, A1529 (1964) there is a degree of arbitrariness in the choice of simplifying assumptions used to obtain numeric values. Those used here can be expressed using Gryzinski's original terminology as follows:

$$
Q_{i j}=\int_{0}^{\infty} v\left(E_{2}\right)\left\{\int_{u_{1}}^{u_{2}} \sigma(\Delta E) d(\Delta E)\right\} d E_{2}
$$

where $I>j$ and $f\left(E_{2}\right)$ is the Maxwell-Boltzmann distribution function for the energies, $E_{2}$, of the free electrons, $u_{1}$ is the energy difference between levels $i$ and $j, u_{2}$ is the energy difference between levels $j$ and $j+1$, and $v$ is the velocity of the electron having energy $E_{2}$. The distribution of bound energies was assumed to be a delta function giving the excited bound electron an energy exactly $E_{1}$ being equal to the ionization potential of the $j^{\text {th }^{2}}$ state. See: M. Gryzinski, Phys. Rev. 115, 374 (1949). Frank-Condon factors are assumed to be unity. 
7. This is equivalent to the collisional radiative recombination rate of Bates et al. (see Ref. 3). The values obtained here agree only to within about a factor of 2 for all temperatures and densities examined owing to the particular choice of approximations used in evaluating the $Q_{i j}$ (see Ref. 6). Those employed here were chosed to give the smallest $Q_{i j}$ in an effort to avoid overestimating the effects of the inclusion of a dissociating level.

8. E. E. Ferguson, F. C. Feh senfeld, and A. L. Schmeltekopf, Bull. Am. Phys. Soc. 10, 187 (1965).

9. G. Herzburg, Molecular Spectra and Molecular Structure (D. Van Nostrand Co., Inc., Princeton, New Jersey, 1950), p. 535-536.

10. The electronic degeneracy of the $n^{\text {th }}$ state of molecular helium is $4 n^{2}$ rather than the hydrogenic $2 n^{2}$. This of course will have no effect on the inelastic and superelastic collision rates between bound levels and since the ionic degeneracy is 2 rather than the hydrogenic unity the rates between bound and free states are similarly unaffected. The only variance arises with the dissociative level in that the discussion of the single dissociative state must be considered to apply literally to a doubly degenerate state instead. With this understanding the theoretical results could be expected to apply to molecular helium.

11. This was reported at the 17th Gaseous Electronics Conference (1965). See: C. B. Collins and W. W. Robertson, Bull. An. Phys. Soc. 10, 189 (1965). 


\section{CAPTIONS}

FIGURE 1 Left: The hypothetical potential curves used in these calculations showing the hydrogenic spacing in energy of the vibrationless ground state of each electronic state.

Right: The equivalent energy level diagram. In this case the third equivalent hydrogenic level has been assumed to be dissociative.

FIGURE 2 The collisional-radiative recombination rate in the absence of a dissociating level as a function of the number of bound quantum levels considered in the calculations for an electron temperature of $250^{\circ} \mathrm{K}$ and a density of $10^{10}$ electrons $/ \mathrm{cm}^{3}$.

FIGURE 3 Calculated recombination rates as functions of electron density: collisional-dissociative rate, $a_{D}$, for various values of $q$, the principal quantum number of the dissociating level; --- collisional-radiative rate, $a_{C R}$, in the absence of a dissociating level. Electron temperatures are: (a) $250^{\circ} \mathrm{K}$; (b) $500^{\circ} \mathrm{K}$; (c) $1000^{\circ} \mathrm{K}$; (d) $2000^{\circ} \mathrm{K}$.

FIGURE 4 Calculated recombination rates as functions of electron density: total electron removal rate, a TOTAL, for various values of $q$, the principal quantum number of the dissociating level; --- collisional-radiative rate, ${ }^{\alpha} \mathrm{CR}$, in the absence of a dissociating level. Electron temperatures are: (a) $250^{\circ} \mathrm{K}$; (b) $500^{\circ} \mathrm{K}$; (c) $1000^{\circ} \mathrm{K}$; (d) $2000^{\circ} \mathrm{K}$.

FIGURE 5 Calculated populations of excited states produced by electron-ion recombination at electron temperatures of $2000^{\circ} \mathrm{K}$ and $10^{13}$ electrons $/ \mathrm{cm}^{3}$. (a) --- populations expected in systems including a dissociating level at $q=4$; populations expected in the absence of dissociating levels; (b) --- populations expected in systems including a dissociating level at $q=6$; _ populations expected in the absence of dissociating levels. 
FIGURE 6 Calculated collisional-dissociative recombination rate as a function of $A_{D}$, the rate coefficient for spontaneous dissociation of the $q^{\text {th }}$ level, and parametrically as a function of $\mathrm{g}$.

FIGURE 7 Calculated total electron removal rate as a function of the effective quantum number at which dissociation is assumed to occur for an electron temperature of $2000^{\circ} \mathrm{K}$ and $10^{13}$ electrons $/ \mathrm{cm}^{3}$. __ results for a non-degenerate dissociating state with continuously varying effective quantum number; - results for a dissociating level with hydrogenic degeneracy and integral quantum numbers. 


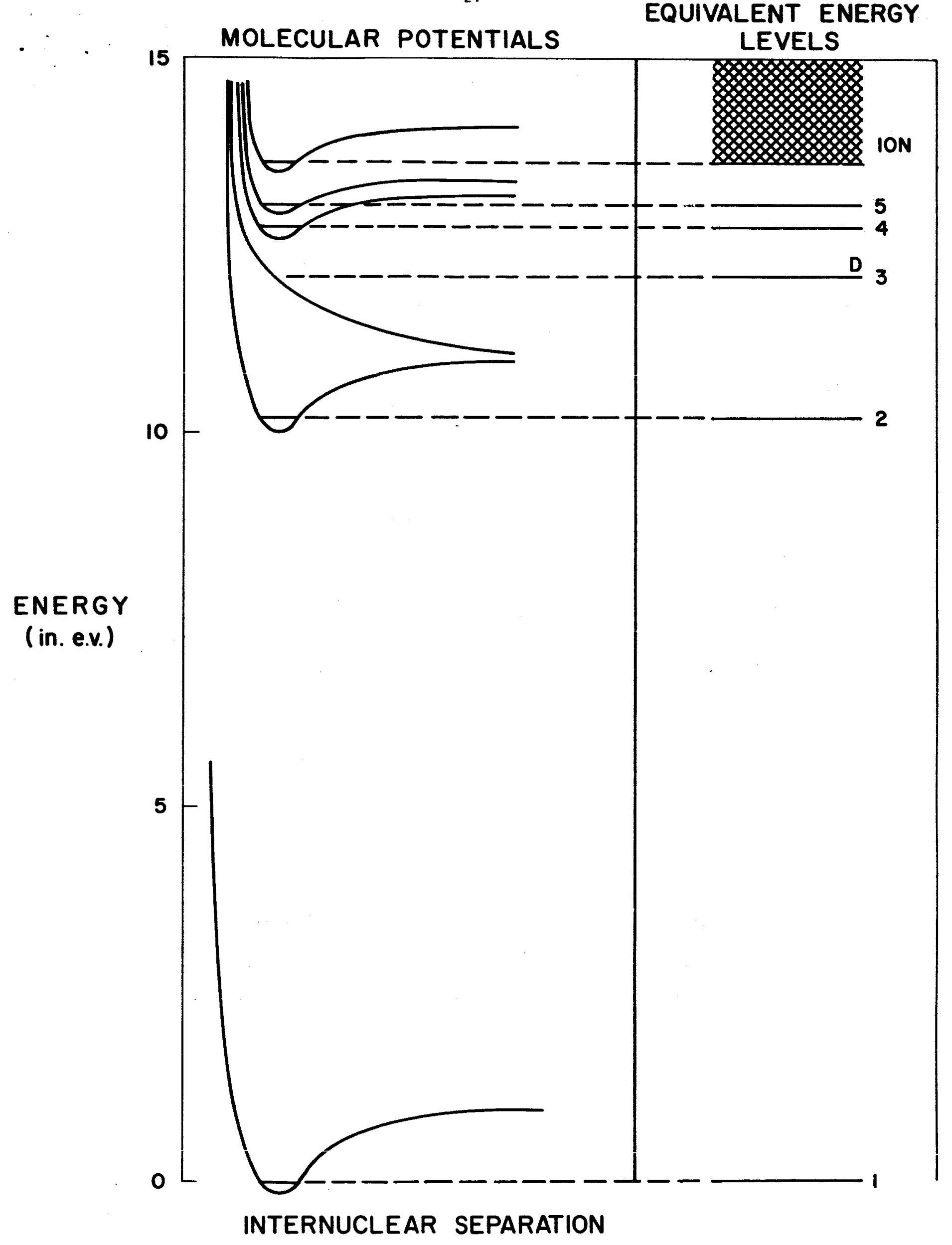

FIGURE I 


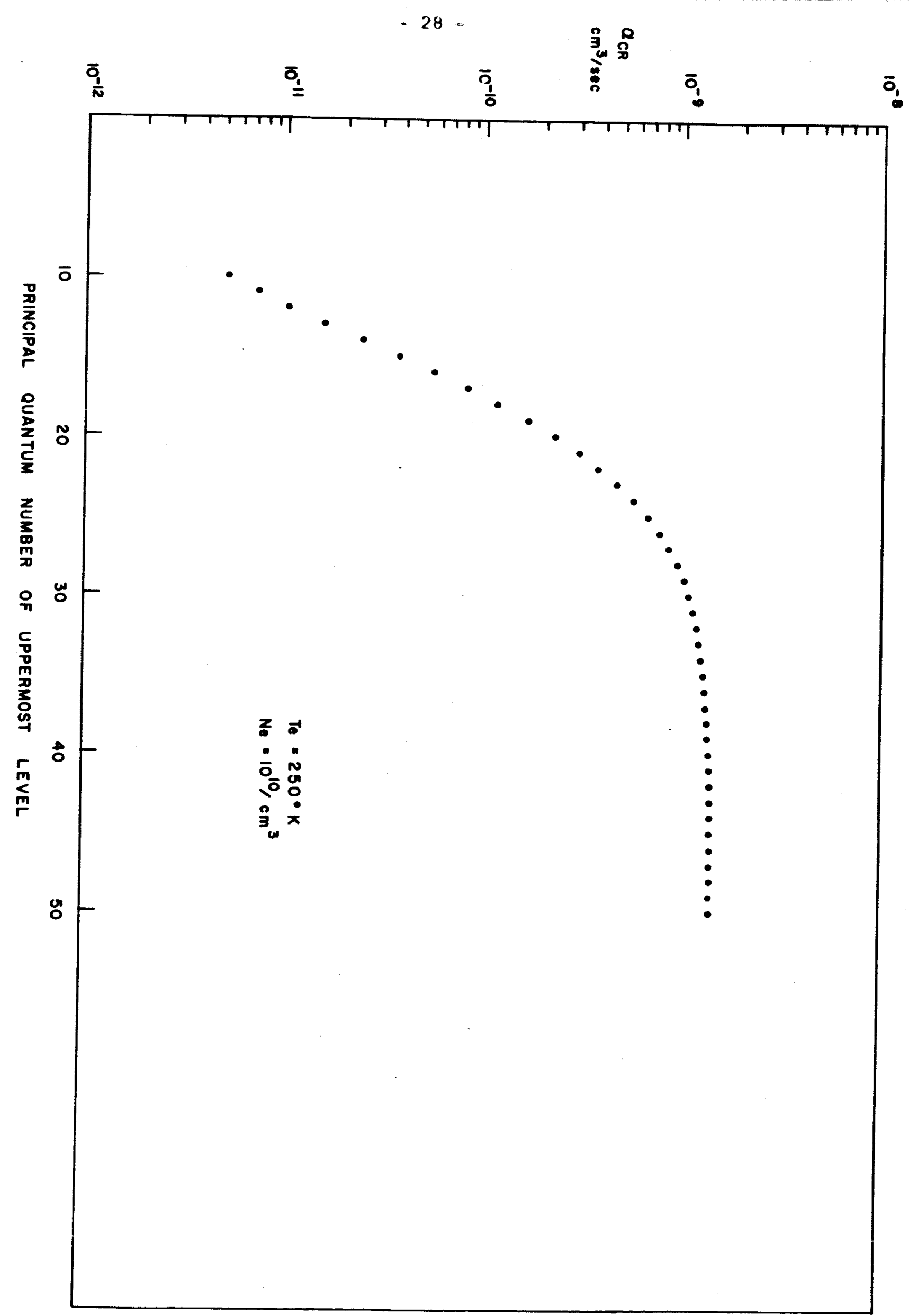

FICUURE 2 


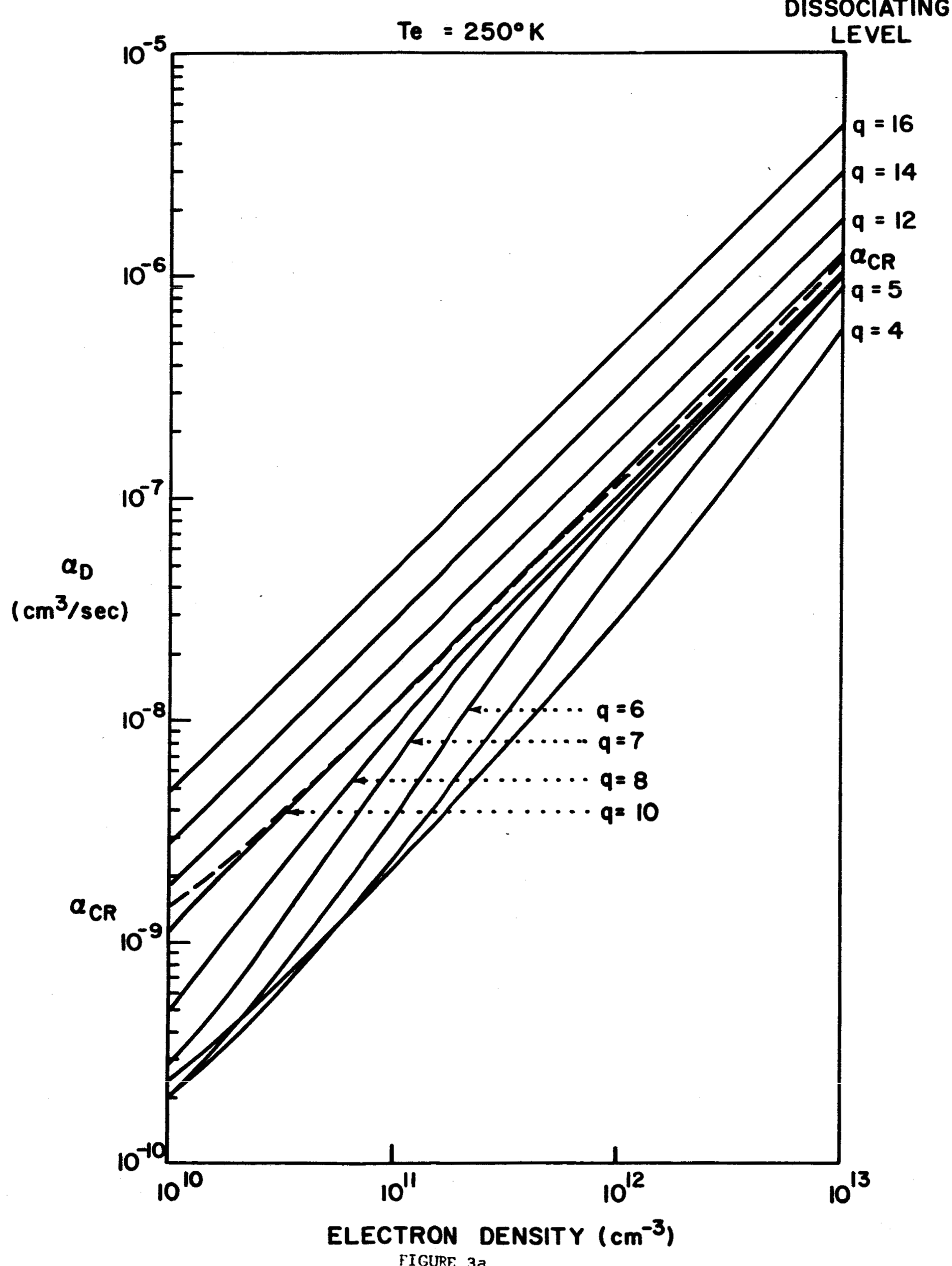




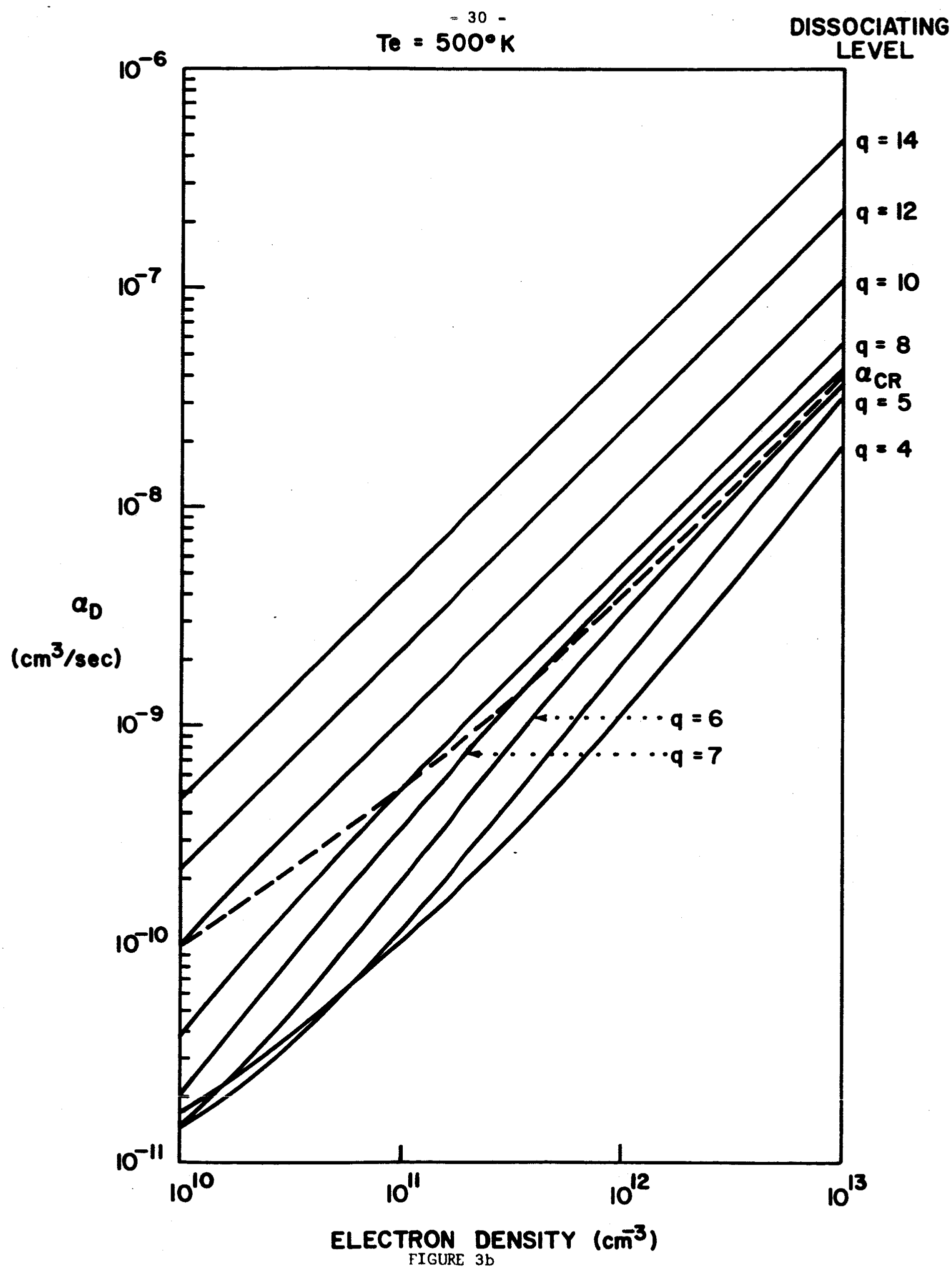




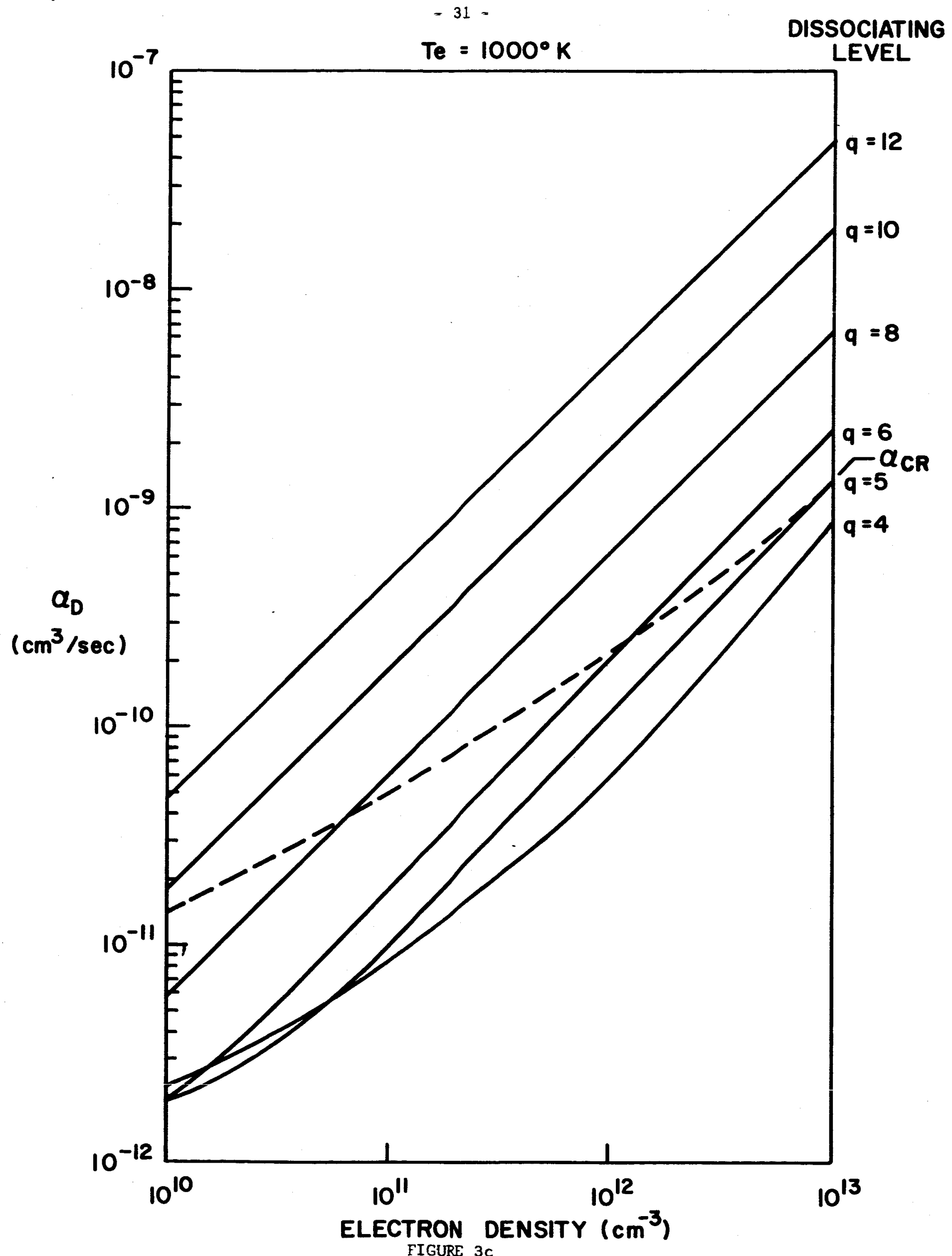




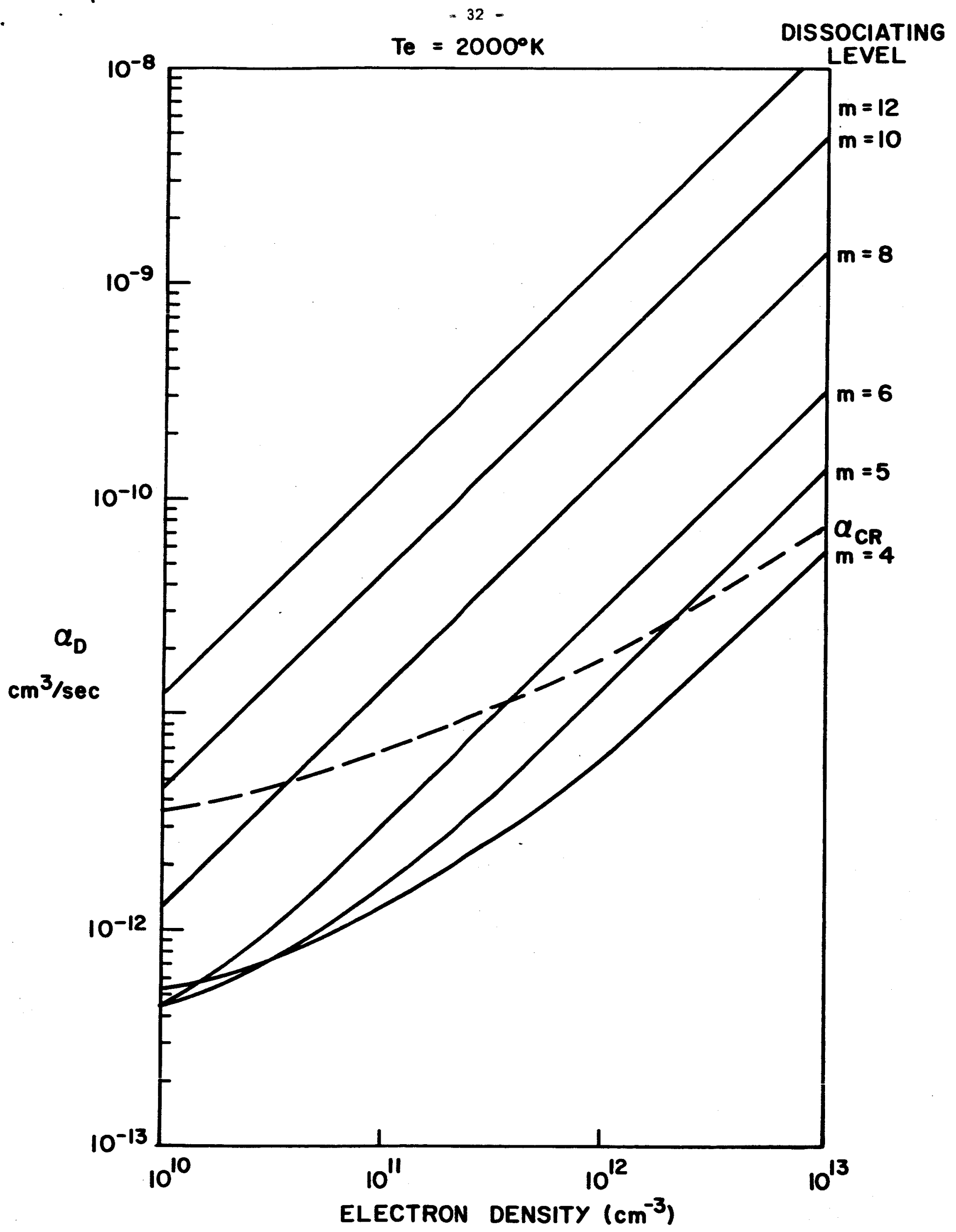




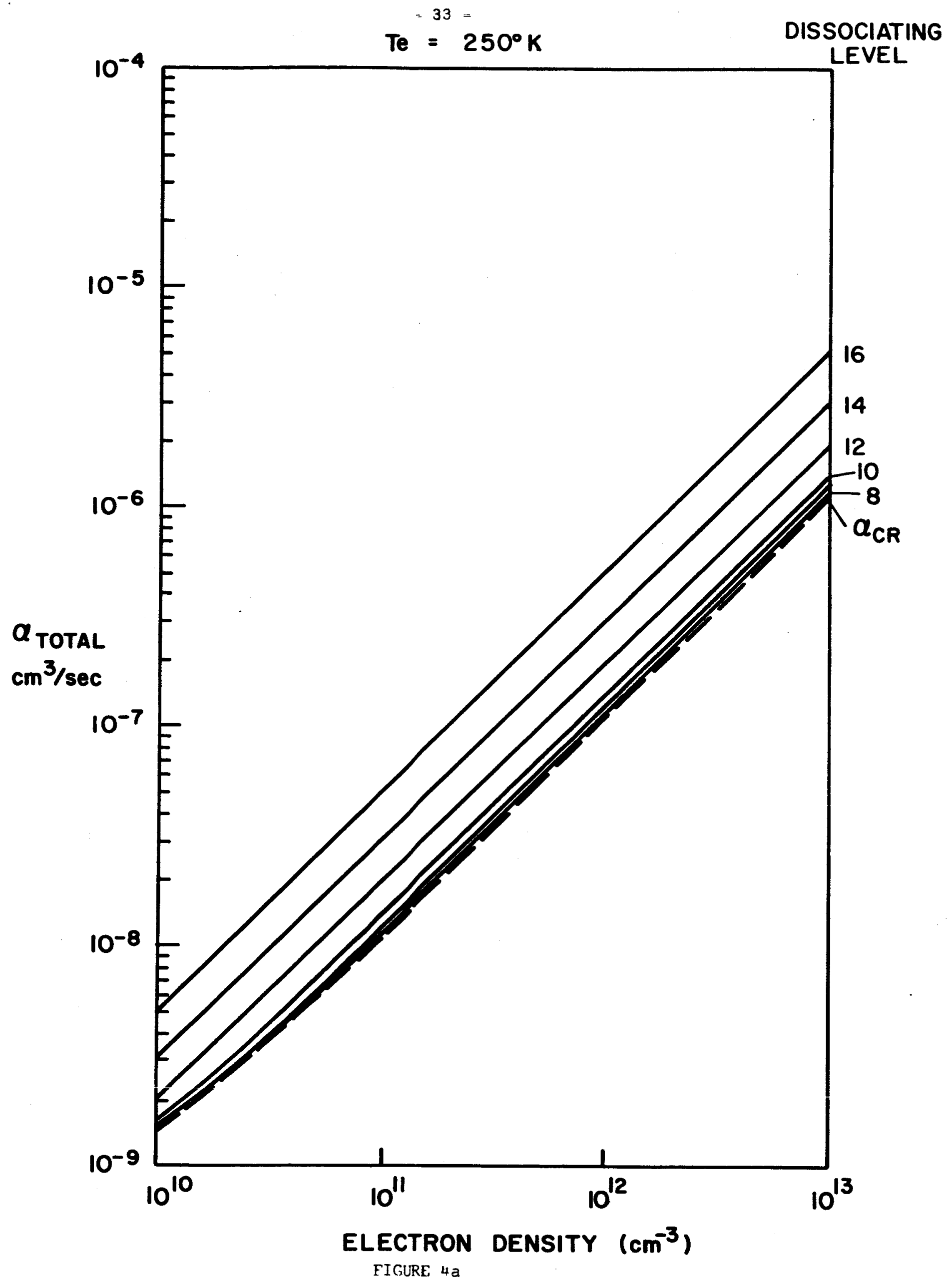




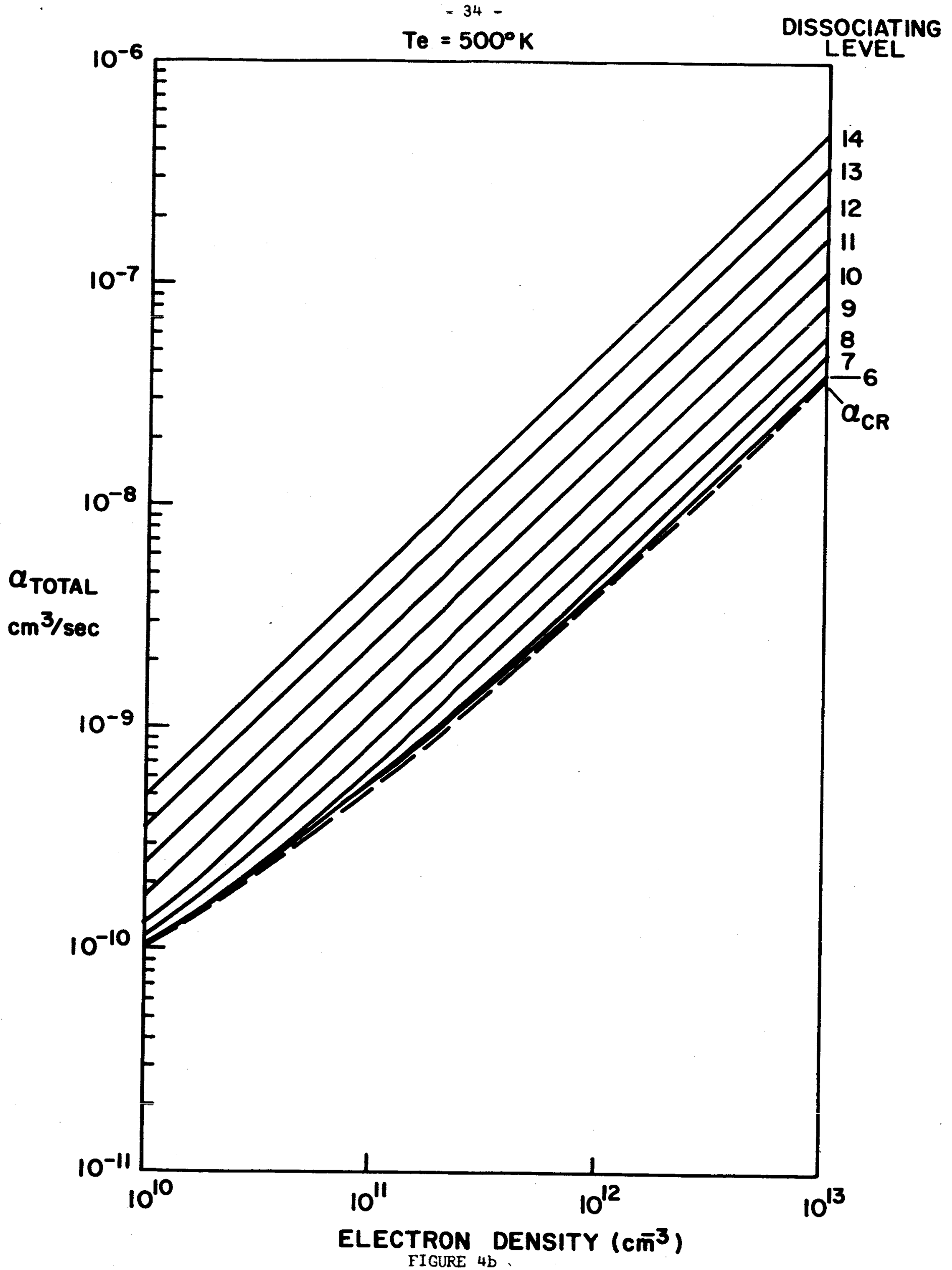




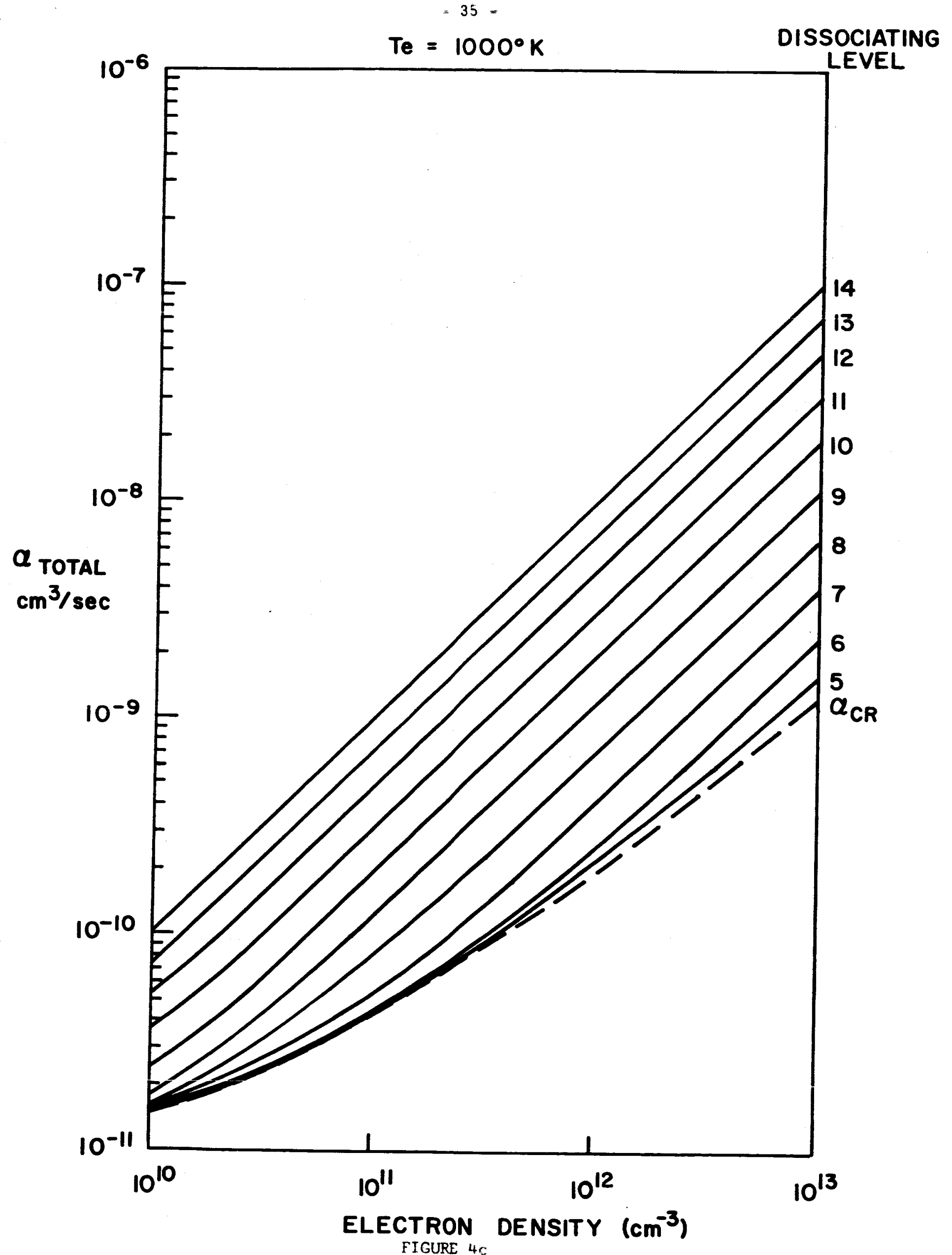




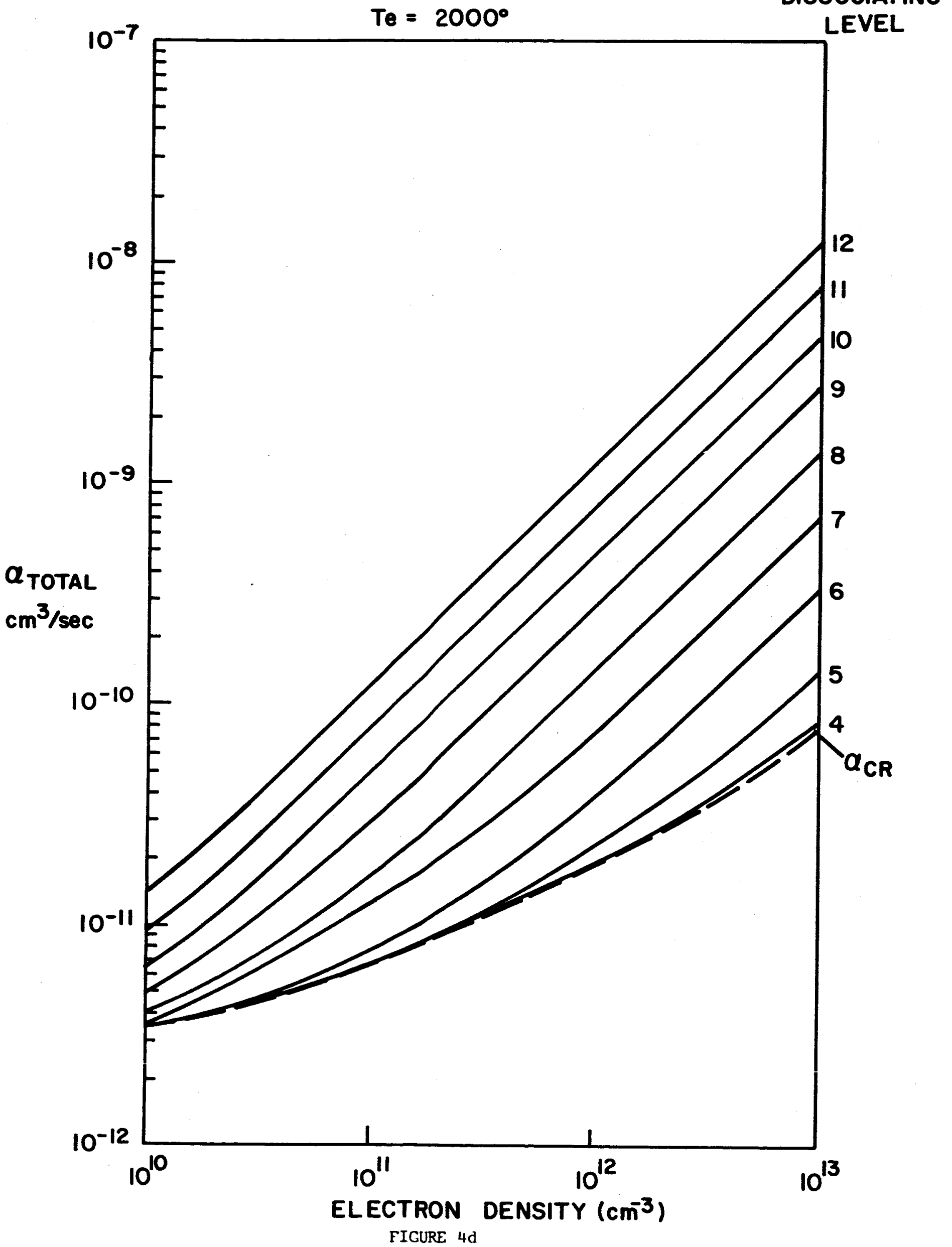




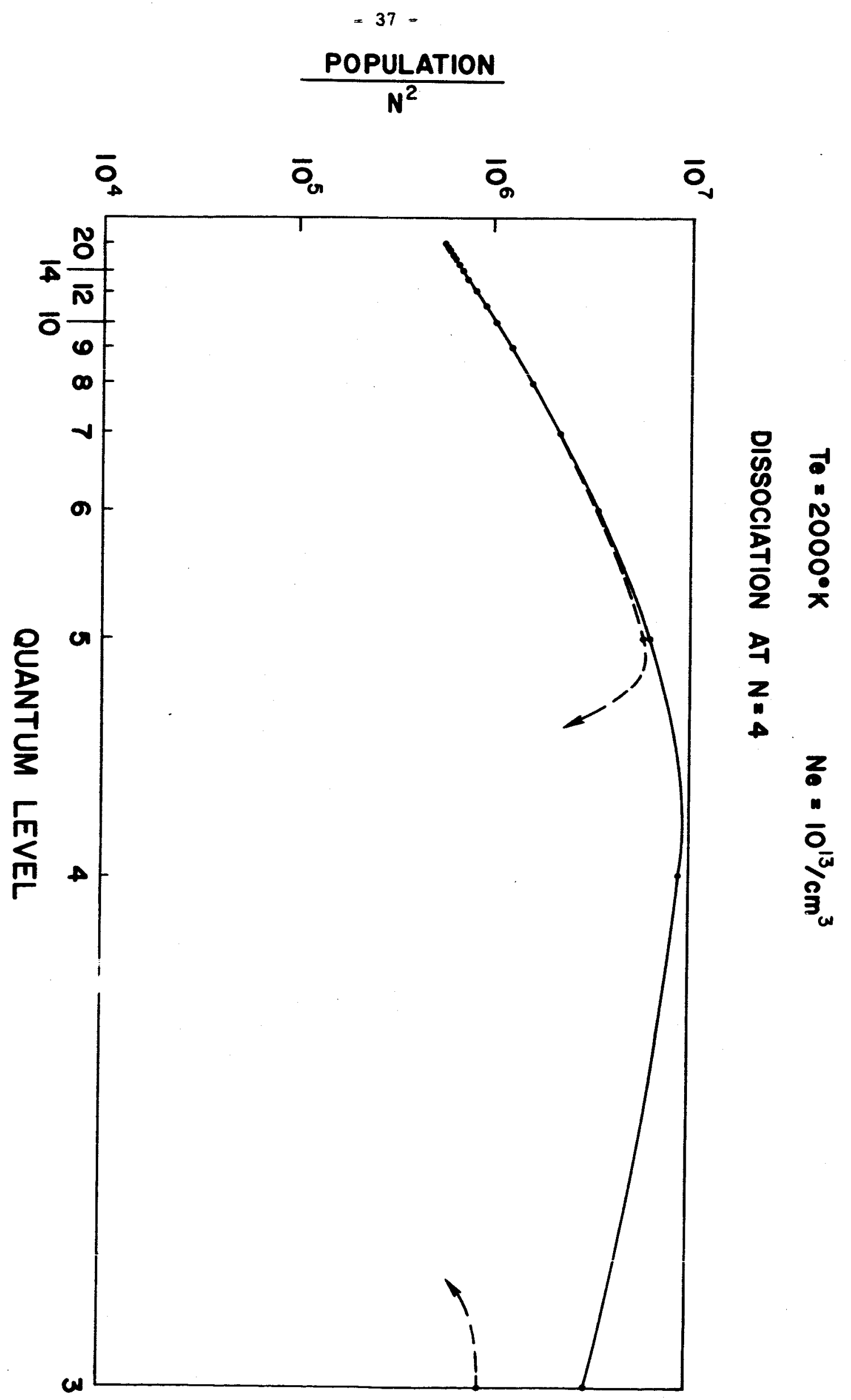

FIGURE $5 a$ 


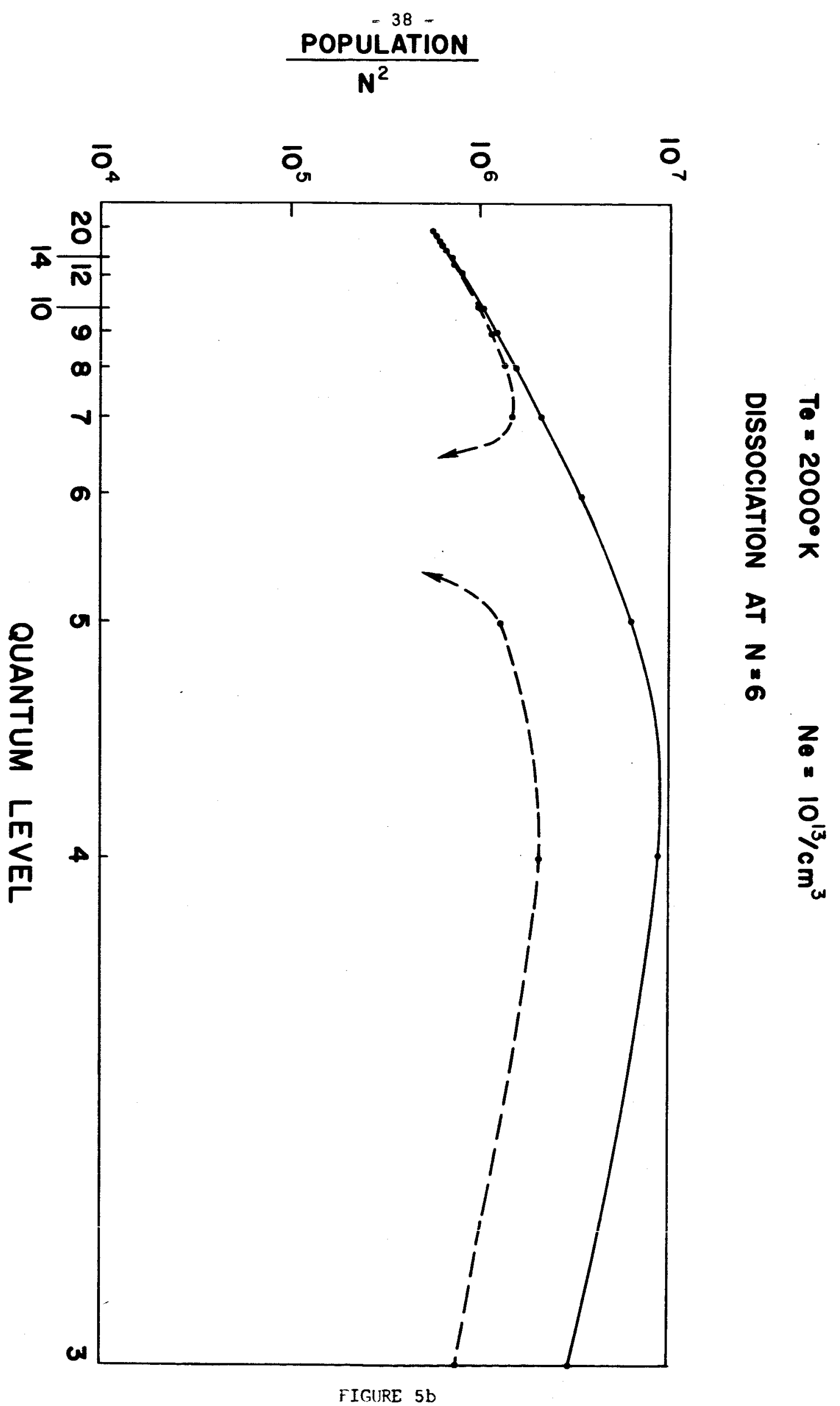




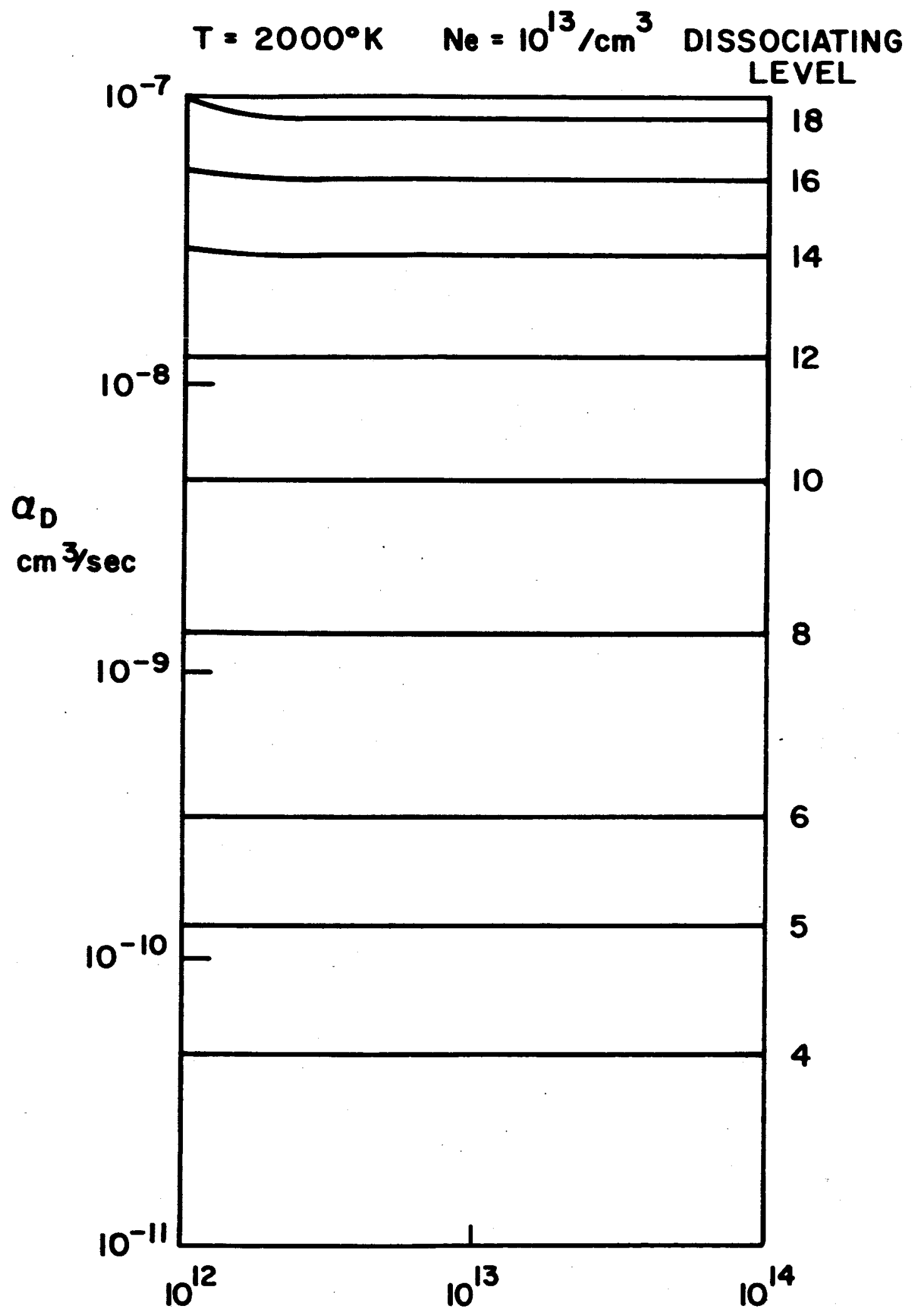

RATE COEFFICIENT FOR SPONTAENOUS DISSOCIATION 


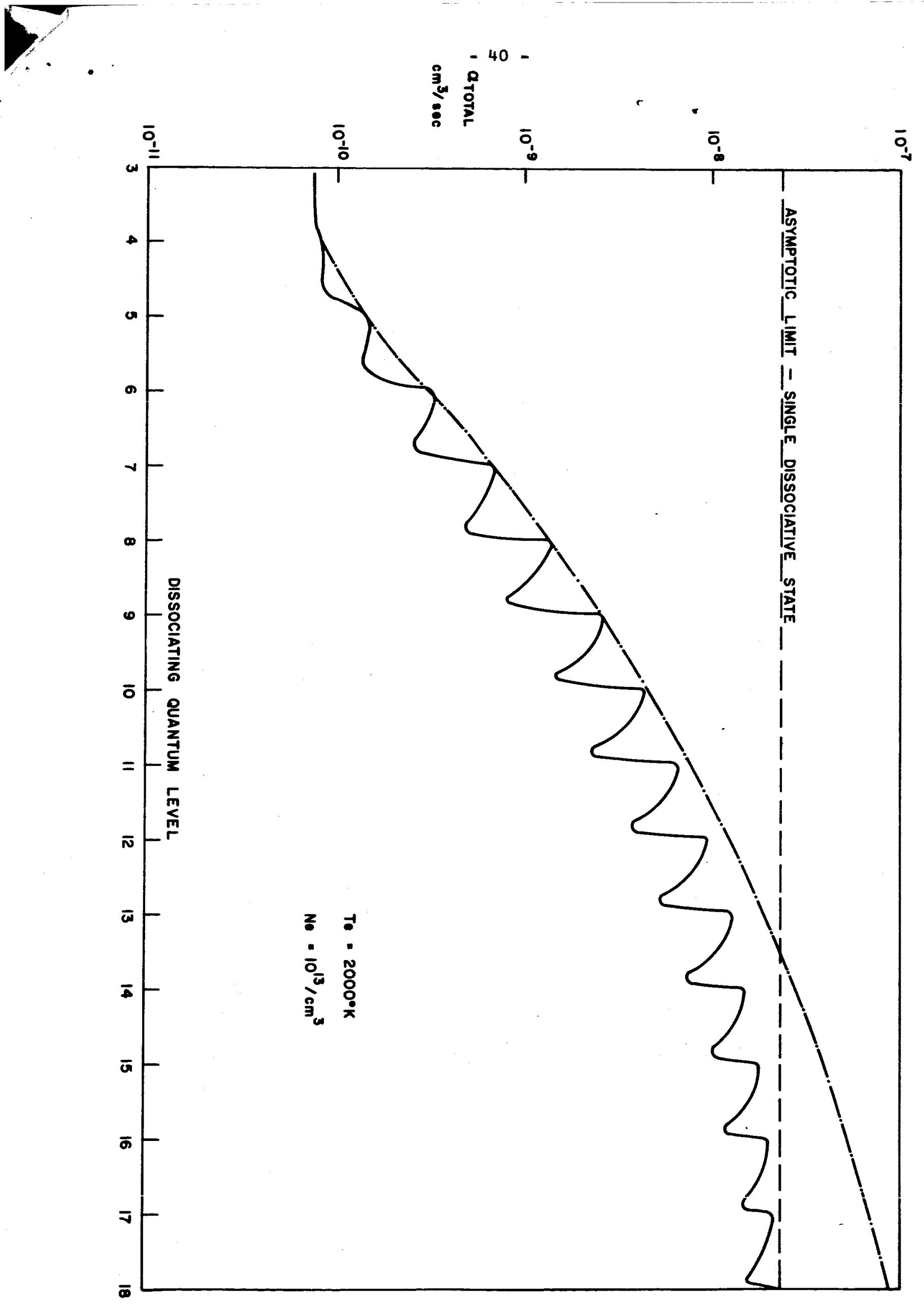

FIGURE 7 